\title{
Catel Muller et Ulli Lust entre BD féminine et BD féministe
}

\author{
Juliette Feyel \\ Cambridge University
}

Qu'entendre par l'expression « bande dessinée au féminin »? D’abord une bande dessinée créée par des femmes. Le phénomène est suffisamment rare pour qu'on s'interroge; en effet comment se fait-il que la profession reste encore si majoritairement masculine ${ }^{1}$ ? Depuis quelques années, les protestations concernant la misogynie du milieu de la bande dessinée prennent un tour médiatique. En 2011 une pétition a récolté près de 3000 signatures pour dénoncer la discrimination à l'embauche de la célèbre maison d'édition américaine DC Comics : «Recrutez plus de femmes; en voici quelques-unes pour vous donner des idées » (suit une longue liste d'illustratrices et de scénaristes) (Collectif, en ligne). Pendant ce temps, le nombre d'événements culturels, de collections réservées aux femmes, d'expositions et de conférences ne cesse de s'amplifier'. Enfin, le prix Artémisia a été créé pour, comme l'affirme Chantal Montellier, «valoriser et éclairer certains albums faits par des femmes [...]. Avec ce prix, on veut à la fois sortir de cette logique et se donner à soi-même la reconnaissance qu'on nous refuse. » (Association Artemisia) On peut qualifier de féministe cette démarche visant à améliorer la visibilité des talents féminins et à promouvoir l'égalité des sexes.

Des polémiques émergent pourtant en ce qui concerne cette forme de «discrimination positive ». La question n'a pas que des enjeux politiques, comme le montrent les billets d'humeur de Tanxxx. Comme d'autres (Groensteen), elle pense que les éditeurs pourraient être tentés de détourner l'étiquette féministe en vue de faire du profit, tout en se cantonnant à des productions commerciales stéréotypées. 147 créatrices de BD se sont d'ailleurs associées pour signer une charte en ligne qui rejette l'étiquette de «BD féminine ». Le label leur apparaît comme une façon d'essentialiser la bande dessinée de femmes et de la maintenir dans une position d'infériorité. Cela a trop souvent pour effet de réduire les $\mathrm{BD}$ de femmes à un genre qui domine le marché éditorial et qui se cantonne aux codes de la « chick lit » ou littérature " girly », alimentant de la sorte le préjugé selon lequel les femmes ne sauraient pas faire de bande dessinée originale (Charte des créatrices de BD contre le sexisme).

\footnotetext{
${ }^{1}$ Les femmes représentent un peu plus de $12 \%$ des auteurs d'après le rapport de Gilles Ratier.

${ }^{2}$ L'exposition « Regards de femmes » s'est déroulée les 20-24 mars 2014 au salon du livre de Paris, suivie par « Héro(ine)s » au Lyon BD Festival (3-27 juin 2015).
} 
Que serait alors une «bande dessinée au féminin » ou dit autrement, faite « à la manière » des femmes? Grâce à quels critères pourrait-on évaluer la féminité d'une œuvre et parler de bande dessinée « féminine » comme on a parlé auparavant d'« écriture féminine » (Cixous) chez les femmes de lettres? Les femmes auraient-elles de nouveaux thèmes à offrir, des thèmes que la bande dessinée n'avait jamais abordés? Un certain regard, une certaine voix, un certain imaginaire?

On s'appuiera donc sur deux exemples : Kiki de Montparnasse de Catel Muller (avec Jean-Louis Bocquet, 2007) et Trop n'est pas assez. d'Ulli Lust (2012). Toutes deux lauréates du prix Artémisia ${ }^{3}$, elles appartiennent de plein droit aux représentantes de la biographie en roman graphique, appelée de plus en plus souvent « bio graphique ». Muller dessine l'histoire d'Alice Prin dite « Kiki », modèle et muse des artistes, chanteuse, danseuse et personnalité mondaine du Montparnasse bohème des années folles. Lust relate l'été de ses dix-sept ans, quand elle est partie avec son amie Edi en Italie, sans argent ni papiers. Deux histoires de femmes (Kiki et Ulli), racontées par des femmes (Muller et Lust). On cherchera au sein de ces deux œuvres des formes d'inscription du féminin dans le médium en posant successivement les questions suivantes : comment les deux auteures envisagent-elles le rapport entre leur identité de femmes et leur pratique artistique? Quelles modifications font-elles subir à la bande dessinée? Intègrent-elles à leur bio graphique une réflexion sur l'identité ou la condition féminine? Proposentelles d'éventuelles perspectives d'émancipation pour les femmes? Et enfin, comment les situer, plus généralement, dans l'histoire de la bande dessinée? On passera le corpus au crible de ces questions afin de déterminer si ces bios graphiques peuvent être considérées comme féministes, féminines, dans quelle acception et jusqu'à quel point.

En se consacrant à la bio-graphique, Muller et Lust cultivent un genre qui, sans être l'apanage exclusif des femmes, est celui qui attire chaque année davantage d'auteures de bandes dessinées. En effet, loin d'une "bédé» dominée par l'action (aventure, western, thriller, science-fiction, superhéros...), écrite par des hommes à l'intention d'un jeune lectorat masculin, le roman graphique, qui n'a pas cessé de se développer depuis les années 1990, s'adresse plutôt à des adultes et n’hésite pas à laisser une plus large place au réalisme. On le reconnaît souvent à son format plus petit que le 48CC (Guilbert). Une histoire peut s'étirer sur près de 400 pages; l'usage du noir et blanc domine; l'utilisation du "gaufrier », découpage en cases régulières, est au service de récits où la psychologie et les sentiments priment sur le mouvement et l'action.

Or, on observe que le développement du roman graphique, et de la bio

${ }^{3}$ Ulli Lust pour Trop n'est pas assez (2011) et Catel Muller pour Ainsi soit Benoîte Groult (2014). 
graphique en particulier, coïncide avec l'intérêt croissant des femmes pour le neuvième art. Il semblerait que l'évolution du médium lui-même ait créé des vocations, car la bio graphique attire chaque année plus d'auteures ${ }^{4}$. Muller a véritablement accédé à la notoriété avec Kiki (2007), Olympe de Gouges (2012) et Ainsi soit Benoîte Groult (2013); elle se consacre donc de plus en plus aux bios graphiques de celles qu'elle appelle les « clandestines de l'Histoire », ces femmes injustement oubliées par la mémoire collective. « Je n'ai pas vécu la guerre, la ségrégation, j'ai la chance d'évoluer dans une société qui me permet de m'exprimer. Mes BD sont pour moi comme des remerciements à celles qui m’ont précédée. »(Berkani) Dans ses interviews, elle affiche sa volonté d'apporter une contribution à l'avancement de la cause des femmes. Dans un autre entretien, elle déclare :

Oui c'est mon petit parcours féministe; ce n'est pas de la revendication au premier degré, elle est un peu déguisée; en parlant de femmes qui ont lutté pour la liberté des femmes, parfois aux dépens de leur vie, comme Olympe, souvent oubliées, je les fais revivre et leur rends hommage, et en même temps cela permet une réflexion sur le sujet. (Reyns-Chikuma)

Elle déplore la sous-représentation des auteures dans la bande dessinée, cite parmi ses modèles Chantal Montellier ou Claire Brétécher et participe à de nombreux événements pour promouvoir la bande dessinée de femmes. Une revue de presse très fournie et consultable sur son blogue personnel insiste beaucoup sur cet engagement.

De son côté, Lust confie à Marc Sobel qu'elle n'a pu se décider à publier une bio graphique personnelle qu'en liant le récit à des préoccupations plus générales, les différences culturelles et les rapports entre les sexes. Elle dit ne pas reconnaître son expérience de la féminité dans ce qu'on montre « dans les films »: "On ne m'a jamais élevée pour que je m'efface devant les hommes. Je n'ai pas grandi timide. Mon père était un homme gentil et très doux, $\mathrm{j}$ 'avais aussi deux sœurs. Je n'ai jamais eu l'impression qu'une femme devait être farouche, qu'elle devait avoir peur ou autres. Alors je me suis dit : “ok, c'est bon. J'ai une raison politique de parler de moi." »" Â propos d'un autre projet autobiographique, elle ajoute : «Ce qui m'intéresse, c'est le point de vue féminin, la vie féminine. La littérature manque encore de voix féminines. Trop de livres sont écrits par des hommes, même quand ce sont des livres sur les femmes, ça reste... de l'invention, vous voyez ce que je veux dire? ${ }^{6}$

\footnotetext{
${ }^{4}$ Après le triomphe de Marjane Satrapi avec Persepolis (2000-2003), d'autres femmes ont abordé la bio graphique : Zeina Abirached, Miriam Katin, Julie Doucet, Florence Cestac, Aurélia Aurita, Annie Goetzinger, Chloé Cruchaudet, Virginie Greinier, Agnès Maupré, Anne Simon et Corinne Maier..

5 «I was not taught to make myself smaller than the man. I did not grow up shy. My father was a very nice and gentle person, and I grew up with two sisters. So I never had the feeling that a woman has to be shy or frightened or all that stuff. So I thought, "ok, it's good. I have a political reason to talk about myself." » (Sobel).

${ }^{6}$ «What I'm interested in is female perspective, female life. There's still a need in literature for the female perspective. Too many books are written by men, even if they tell about
} 
Ces auteures revendiquent donc toutes les deux, à un moment donné, une forme de féminisme. Mais ce parti pris se manifeste-t-il dans leur façon de traiter le médium graphique et, si oui, sous quelle forme? Pour y répondre, évoquons tout d'abord la façon dont Muller et Lust campent leurs héroïnes. On a beaucoup critiqué la façon dont les personnages féminins sont représentés par la bande dessinée masculine : asexuées comme Bécassine, Adèle Blanc-Sec et Yoko Tsuno, ou au contraire hypersexualisées comme Arya ou Natacha... ${ }^{7}$ Rien de tel chez Muller et Lust qui abordent les défauts physiques de leurs personnages. Ulli se trouve trop ronde et se juge moins séduisante qu'Edi, qui exhibe ses jambes longues et minces en toutes circonstances. Le corps de Kiki évolue avec son âge, elle grossit, vieillit. Si les héroïnes apparaissent nues, les cadrages et les poses sont moins destinés à émoustiller le lecteur qu'à évoquer leur émancipation sexuelle. Non pas bande dessinée érotique, mais bande dessinée sur la sexualité féminine. Ces héroïnes aspirent à la liberté de tout vivre et de tout essayer. Kiki séduit, car, sans peur et prompte à l'emportement, elle se conduit à chaque page comme une insoumise qu'aucune règle conventionnelle n'effraie. C'est en la voyant aux prises avec un serveur méprisant que Man Ray tombe aussitôt amoureux d'elle. De son côté, Ulli rejette tous les modèles que sa famille souhaiterait la voir endosser, étudier ou travailler. Son idéal est de mener une vie de mendicité, d'expédients et de nuits à la belle étoile, car, pour elle, la rue vaut mieux qu'une vie étriquée de petite bourgeoise. Ces héroïnes sont donc à la fois plus réelles, moins idéalisées et animées par un fort désir d'indépendance.

Les deux bios graphiques s'aventurent sur le terrain d'une réflexion sociale liée à la condition féminine lorsqu'elles traitent de la marginalisation qui frappe les femmes qui refusent de se soumettre aux normes. Kiki est chassée par sa mère quand celle-ci découvre que sa fille pose pour un sculpteur. Elle est haïe par ses cousines par alliance, qui l'éloignent de sa grand-mère bien-aimée, car «Pour les gens, être modèle c'est encore plus bas que d'être une putain!» (89) À cause de ses mœurs, on va jusqu'à l'enfermer, la traiter comme une criminelle ou une folle $(260,342)$. Dans Trop n'est pas assez, Ulli découvre pas à pas la terrible violence symbolique et physique infligée aux femmes qui ne sont rattachées à rien. Elle est insultée par les passants à Vienne quand elle fait la manche, en Italie elle est en butte au désir des tous les hommes qui passent et subit une série d'agressions sexuelles de plus en plus graves. Les péripéties de son voyage lui révèlent d'abord que, dans une société aussi fortement patriarcale qu'en Sicile, une femme ne peut survivre seule, il faut qu'un homme soit là pour éloigner les autres (261). L'exemple d'Edi montre de manière éclatante que le protecteur ne tarde jamais à se transformer en souteneur.

women, they are still... inventions, you know ?» (Sobel)

${ }^{7}$ Voir aussi le projet « Héro(ine)s $»$. 
En outre, les deux héroïnes sont fréquemment aux prises avec un phénomène typiquement féminin, l'aliénation et la réification de leur corps par le regard des hommes. Kiki apprend très vite que ses charmes seront sa meilleure monnaie d'échange pour survivre. Mais elle veut pouvoir en vivre sans pour autant se prostituer et être sous l'emprise d'un maquereau qui la battrait (83). Son grand nez l'empêche d'être recrutée dans un cabaret (84), mais sa beauté non conventionnelle attire l'œil des peintres modernes (94), qui lui ouvrent bientôt les portes d'un destin extraordinaire. Or, à force de poser pour des œuvres d'avant-garde où on ne la reconnait pas, elle finit par trouver que ces hommes utilisent son corps à des fins personnelles qui ne concernent ni ce qu'elle est ni son mérite personnel :

Man Ray - Mais Kiki, les personnages de ce film ne sont que des marionnettes. Ça n'a rien à voir avec toi ou moil Toi, tu es l'objet de mon affection! Kiki - OBJET! Voilà! Je n'existe pas pour toi. Je suis une FORME. Une forme de femme... ABSTRAITE!

Man Ray - Où vas-tu?

Kiki - Plonger dans la réalité! (288)

Elle croit un temps se réapproprier son image en essayant le cinéma, mais la déception est la même; aux studios Astoria de la Paramount, les actrices sont exploitées à l'échelle industrielle (188-191). Une scène pathétique la montre surgissant à un vernissage de Kisling en 1951. Ceux qui la célébraient jadis peinent ou rechignent à reconnaître cette femme obèse qui réclame à boire : «Vous connaissez cette clocharde? » demande-t-on au peintre (357). Muse, modèle, égérie, le statut de Kiki ne dépend que du regard d'autrui. Sa beauté lui a ouvert des horizons insoupçonnés, mais, à partir du moment où elle se fane, les artistes se tournent vers d'autres modèles, d'autres « outils de travail » (104) selon les mots de Kisling.

Afin de se soustraire à l'attention des hommes, Ulli cache son corps sous des tee-shirts informes. En vain : «Peu importe à quoi je ressemble ou ce que je peux dire. Je ne suis qu'un trou sur deux jambes, avec des seins qui pendent devant. Oui, voilà ce que je suis. Et une fois que le trou est couché, n'importe qui peut s'y fourrer.» (283) Épuisée par les avances qu'elle doit constamment repousser, elle rêve avec nostalgie du temps de l'enfance sexuellement indifférenciée (249-250). Elle tente de s'enlaidir en se laissant grossir, mais, ironie du sort, les critères changent d'un pays à l'autre et l'effet produit est l'inverse de celui qu'elle cherchait: «Un vrai visage d'ange... [regardant Ulli] Contrairement à celle-là... [désignant Edi] les hommes du sud aiment les filles plus rondes. Eh bien quoi? T'as 17 ans, t'es blonde aux yeux bleus et tes seins ont encore grossi. » (315)

Où qu'elle aille, Ulli sent qu'elle n'est qu'un objet sexuel chez qui la manifestation d'un libre arbitre est perçue au mieux comme une bizarrerie, au pire comme un affront devant être châtié. Une table des valeurs radicalement différente l'oppose aux Siciliens qu'elle côtoie. Si elle proteste 
parce qu'on l'a insultée, on lui répond que refuser d'obéir aux hommes est non seulement un affront envers leur virilité, mais aussi un déshonneur pour sa propre féminité $(271,378)$.

Ces auteures renouvellent ainsi la bande dessinée grâce à la complexité qu'elles confèrent à des héroïnes placées dans des contextes sociaux bien précis. Elles font entrer de nouveaux thèmes dans le roman graphique et participent en même temps à la constitution d'une histoire des femmes à travers les destins de celles qui ont tenté d'échapper aux normes imposées à leur sexe. Elles suscitent une réflexion sur l'aliénation des femmes, sur le rôle qui leur est prescrit et sur les menaces auxquels elles s'exposent si elles refusent de jouer le jeu. En cela, il faut reconnaître l'engagement féministe de Lust et Muller. Or, on pourrait opérer une distinction entre une posture que l'on pourrait qualifier de " féministe », réactive, contestataire, qui critique les normes patriarcales, et une posture "féminine», affirmatrice d'identité. Dès lors, où situer Lust et Muller? Ces deux récits proposent-ils des stratégies d'émancipation? La question est posée du point de vue actanciel. Kiki et Ulli réussissent-elles à atteindre leur but, à savoir passer du statut d'objet passif exploité par les hommes à celui de sujet?

Trop n'est pas assez reprend nombre de traits du roman picaresque dans sa structure narrative. Une héroïne partie de rien se confronte en voyage à une série de rencontres et de mésaventures, ce qui fournit un prétexte pour peindre un tableau de mœurs. Les interventions de l'auteure, les extraits de son journal d'alors, ses commentaires en voix off, accompagnent le récit comme la voix du picaro, narrateur rétrospectif et distancié. Ulli passe de la naïveté à une lucidité supérieure. Au début, elle croit accomplir un acte de rébellion radical en devenant une "freak», c'est-à-dire l'une de ces jeunes qui choisissent de vivre dans la rue par conviction anarchiste. Pour le repris de justice, au contraire, il est absurde de chercher à se déclasser volontairement :

Fred — Qu'est-ce que tu fous ici? Tu pourrais faire quelque chose d'intelligent...

Ulli — Je fais quelque chose d'intelligent! Je veux dire, je refuse cette société de merde! Je la quitte!

Fred - (Il s'esclaffe longuement). Mes petites, vous êtes juste descendues dans les bas-fonds! (45)

La vision romantique d'Ulli se lit encore dans cette conversation :

Ulli - Que ferais-tu de ton temps si tu savais qu'aujourd'hui c'est le dernier jour de ta $v^{8} e^{8}$ ?

Edi — Je me foutrais de tout! Je ne me laisserais plus emmerder, j'aurais plus peur de rien, et... je ne ferais que ce qui me plait vraiment! WOW!

Ulli — T'as pigé!

Ensemble - Ha ha ha ha ha ha (71)

Mais l'histoire se clôt sur une désillusion amère : «J'ai été violée... Ce n’était

${ }^{8}$ Cette expression est le titre de l'édition originale, Heute ist der letzte tag vom rest deines lebens. 
pas ce qu'il y avait de pire. Le pire... c'est d'être traitée comme un petit toutou, qui par hasard sait parler. Mais ce que toutou dit, tout le monde s'en fiche. » (425). Ulli ne souhaite plus rien d'autre que de rentrer chez elle et ce départ apparaît à Andreas comme une démission.

Andreas — Pourquoi tu ne m'as rien dit? Pourquoi tu fais ça?

Ulli - Ecoute, je ne sais plus ce que je fous encore là.

Andreas - (vexé) Alors va-t'en. Je pensais que tu étais plus aguerrie. T’es qu'une midinette, comme ta copine, cette lâche.

Ulli - Pas vrai!

Andreas - Rentre chez toi, va te fourrer dans le nid douillet de papa et maman.

Ulli - Conneries! (439)

Le retour dans le giron familial est sanctionné par le silence des parents, les réprimandes de la grand-mère et la prise d'un bain, sorte d'ablution purificatrice pour accéder au sanctuaire de la maison (448-449). Cependant, à la dernière page, Ulli qui s'était d'abord couchée dans le lit, préfère s'endormir à même le sol de la chambre (450). Comment interpréter ce final? Serait-ce une façon pour l'héroïne de maintenir son anticonformisme, cette fois sur un mode mineur, moins frontal, moins provocateur? Mûrie par ses aventures, Ulli aurait-elle décidé de poursuivre désormais la lutte sur un terrain plus symbolique? Ce terrain pourrait être justement celui sur lequel s'étend ce long roman graphique. Le récit des désillusions offrirait à Lust devenue auteure l'occasion de se réapproprier de manière cathartique son passé. Si les événements qu'elle raconte sont parfois terribles, Lust ne rechigne pas à les mêler d'humour (274-277). Elle peut apporter sa propre version de l'histoire aux dépens de ceux qui, comme Edi, ont tenté de rejeter toutes les fautes sur elle; elle peut réaffirmer son moi : « je suis encore libre, et debout! Contrairement à Edi... [...] Invincible comme Siegfried après avoir baigné dans le sang du dragon - voilà comment je me sentais. » (415-416) Les paradoxes du récit de soi permettent à Lust de mener le récit à deux niveaux, d'un côté la jeune et naïve Ulli, de l'autre le regard à la fois moqueur et bienveillant de la narratrice qui rattrape les erreurs de son héroïne en insistant sur l'affirmation de sa liberté.

En revanche, la vie d'Alice Prin est racontée par Muller et Bocquet du jour de sa naissance jusqu'à son enterrement. Les premiers chapitres associent très fortement le destin de l'héroïne à une logique œdipienne. Son esprit de révolte aurait trouvé son terreau dans un double refus d'amour, celui du père, figure d'autorité qu'elle n'aura de cesse de défier, mais aussi de rechercher à travers les autres hommes de sa vie (33-34), puis celui de la mère («Tu n'es qu'une ignoble putain! Tu n’es plus ma fille! », p. 75), celle qui lui aura "toujours manqué », dit Kiki (320). Cela expliquerait pour les deux auteurs pourquoi l'amour aurait toujours été la grande affaire de sa vie.

Kiki - Que la vie est belle! [...] Parce qu'il y a du soleil...

Treize - C'est suffisant à ton bonheur?

Kiki - ... il me faut de l'amour aussi... beaucoup d'amour! (207)

Or, cette vie se déroule également sous le signe de l'alcool : «Moi, j’ai 
commencé à biberonner avant ma première tétée! » (294); elle boit quand elle danse sur les tables à dix ans et jusqu'à son dernier souffle. Kiki est ainsi présentée comme souffrant d'un perpétuel manque à être, un déficit narcissique que ni le regard de ses amants, ni celui du public ni même celui des peintres ne parviennent à combler. Si elle prend de la drogue, c'est « pour ne pas devenir folle d'ennui»(342). Une vie de fêtes perpétuelles a transformé la Reine de Montparnasse en épave solitaire que le vin détruit lentement. À la fin, rien ne nous est caché ni des coliques ni des vomissements dans lesquels elle trouve finalement la mort (365-366). Kiki apparait-elle comme ayant accédé au statut de sujet? Rien n'est moins sûr. Dans l'épisode de Marseille, les lettres d'Aragon et de Desnos destinées à certifier son statut d'artiste ne sont d'aucun poids face à l'institution judiciaire; ce n'est qu'en plaidant des troubles mentaux qu'elle parviendra à éviter la prison (263-264). Même le succès qu'elle remporte en tant que peintre est entaché d'ambiguité. On lui dit, non sans condescendance : « [ceux qui achètent vos peintures] sont sensibles à la fraîcheur de votre art... À son innocence! » (274) Kiki est-elle une véritable artiste comme ses amis ne cessent de le lui répéter? Au cours d'une dispute, elle jette un encrier sur le mur. «Magnifique! » s'exclame Ray dont le regard, tel celui de Marcel Duchamp sur un ready-made, transforme la tâche en œuvre :

Man Ray - Regarde comme c'est beau ce que tu as fait Kiki...

Kiki - Je ne l'ai pas fait exprès. .. C'est ta tête que je visais.

Man Ray - C'est pour ça qu'il y a tellement de rage et de force dans cette tâche. Tu vois, le moindre de tes mouvements peut être un moment de l'histoire de l'Art. » (204)

Le statut d'artiste de Kiki lui est décerné par les hommes, comme si « ce côté naturel... animal des champs » (209) ne pouvait être révélé que par un regard masculin. La dernière image du livre montre Man Ray dans son atelier, entouré des portraits les plus célèbres de Kiki (368). En repoussant les journalistes qui veulent les acheter, il les désigne en disant «mes » photos (367). C'est que le véritable sujet de l'œuvre d'art est moins le modèle passif qui pose que l'artiste actif qui crée la photo. Dépossédée de son image jusqu'au bout, Kiki resterait finalement avant tout mémorable en tant que chef-d'œuvre de Man Ray. Catel Muller a certes voulu faire un geste féministe en rendant hommage à une femme qui a bravé les conventions de son temps. Kiki ressort toutefois de cette bio graphique comme une demimondaine ayant bénéficié de circonstances exceptionnelles pour se faire un nom, à tout jamais immortalisée comme violon d'Ingres du photographe, métonymie du Montparnasse des années vingt.

Enfin, il s'agit de préciser la place qu'occupent ces deux auteures dans le paysage de la création graphique en général? L'enjeu est de taille puisque le soupçon pèse semble peser sur la capacité des femmes à produire une bande dessinée de qualité. Si l'on cherche à promouvoir les créatrices de $\mathrm{BD}$, si l'on veut vraiment qu'elles soient reconnues comme artistes à part entière, débarrassées des clichés « jeunesse » et « girly», il faut que les auteures se 
montrent capables de rivaliser avec les hommes.

La naïveté du trait a été remise au goût du jour par l'expansion du roman graphique. Le graphisme naï de Catel Muller reprend la ligne claire développée dans des travaux d'illustration antérieurs en littérature jeunesse et en $\mathrm{BD}$ « chick lit » ». Il ne faut pas pour autant occulter le fait que sa charte graphique ${ }^{9}$ relève du réalisme le plus strict. La technique du noir et blanc est travaillée comme on pourrait le voir sur une photo. Les aplats de noir et les hachures représentent les ombres et les couleurs foncées. Les mouvements sont rares, les scènes de bagarres elles-mêmes reflètent une singulière immobilité. On pourrait l'expliquer par la méthode de travail de Muller et Bocquet, telle qu'ils l'ont décrite dans un entretien pour Paris Match (Lestavel). Ils passent beaucoup de temps à réunir biographies, témoignages, documents d'archives et photographies. Une longue bibliographie est fournie en fin de volume, preuve du soin particulier que les deux auteurs attachent à l'authenticité de leurs informations. Les lieux ont été parfois croqués directement sur le vif, les visages des célébrités ont été dessinés d'après des photos. On a donc affaire à une reconstitution historique du même type que les docufictions, où une intrigue lie ensemble une multitude de documents qui ont été redessinés afin de se fondre harmonieusement dans l'ensemble.

\footnotetext{
${ }^{9}$ Par « charte graphique », expression reprise à Catel Muller elle-même, on entend non seulement le style de graphisme, la facture du trait, mais aussi le contrat de lecture sur lequel il s'appuie.
} 


\section{CATEL \& BOCQUET}

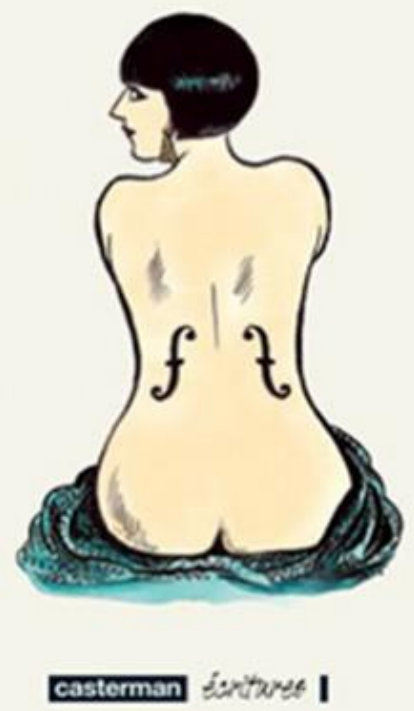

\section{Couverture}

Les planches se présentent comme une suite de dialogues avec peu de mouvements, une nette prédominance du plan rapproché et du montage champ/contrechamp. Autrement dit, l'essentiel de la narration est porté par le texte, la fonction principale du dessin dans l'intrigue étant d'attribuer aux personnages leurs répliques. Bocquet définit d'ailleurs la bande dessinée comme « faite de dialogues et d'ellipses » (Lestavel). C'est peut-être à cause de cette conception bien particulière (et quelque peu réductrice) de la bande dessinée qu'il a finalement laissé assez peu de liberté d'expression à l'illustratrice. Rares en effet sont les passages silencieux. On obtient donc avec Kiki une bande dessinée où le texte prime sur l'image et où les archives priment sur les expérimentations graphiques. La représentation de l'héroïne serait ainsi doublement subordonnée au «male gaze» dont parle Laura Mulvey (1975), perspective du photographe qui regarde son modèle, perspective du scénariste qui fait parler son personnage de papier.

Lust a elle aussi recours à un style "naif », mais le rapport entre texte et images est conçu différemment. L'auteure se repose davantage sur le pouvoir signifiant des images pour raconter son histoire. Utilisant la caricature, la 
dessinatrice a donné aux personnages la tête de leur emploi dramatique. Ils ne sont en fait pas réalistes, ils sont présentés tels que l’héroïne les perçoit.

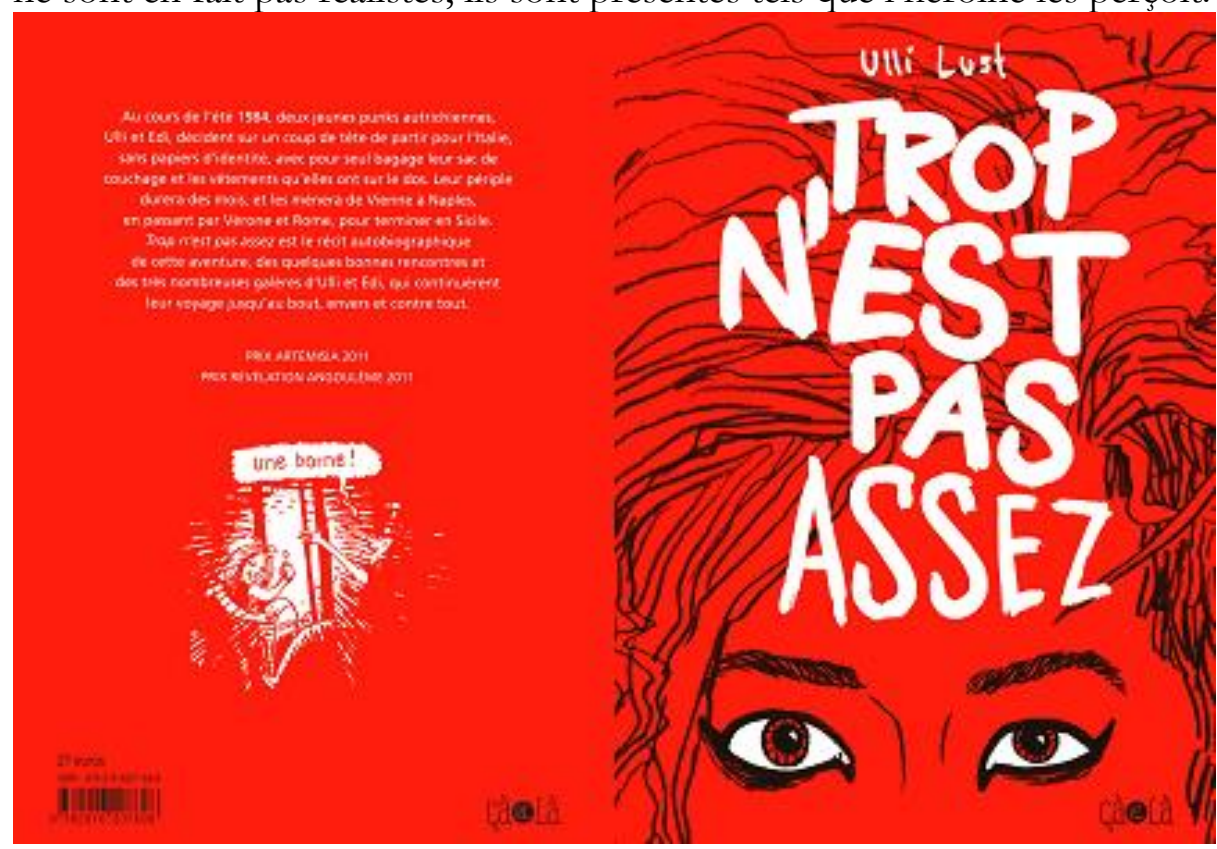

Couverture

On assiste donc parfois à une irruption du fantastique dans la trame du récit comme lorsque, animée d'un sentiment de liberté et de bien-être, Ulli s'envole au-dessus de Rome et nage dans les airs en cabriolant (172-173). Pour exprimer la peur, le soulagement ou la colère, la dessinatrice reprend des symboles graphiques traditionnels, gouttes de sueur, petits nuages sortant de la bouche ou du nez des personnages. Des polices différentes distinguent l'allemand et l'italien. L'anglais est restitué tel quel. Le sicilien, qu'Ulli ne comprend pas, est transcrit sous forme de gribouillis continus. Le même objectif est atteint par les cadrages en plan subjectif (369), quand les hommes qui entourent Ulli l'observent avec convoitise. Une vue de la maison de haut (373) permet au lecteur de mesurer le degré de confinement dans lequel se trouve l'héroïne et sa sensation d'être prise au piège. Au fur et à mesure que l'histoire évolue, la fréquence des audaces graphiques augmente. Afin d'évoquer l'expérience du harcèlement, Lust est même amenée à inventer ses propres symboles graphiques. Elle dessine par exemple des mains qui jaillissent des yeux des hommes pour agripper le corps d'Ulli (226). Son sentiment de vulnérabilité est métaphorisé dans une case où elle apparaît minuscule et encerclée d'ombres géantes, mi-animales, mi-humaines (245). Le danger, la violence ou l'agressivité sont représentés par de grandes formes noires et hirsutes qui s'abattent sur l'héroïne (380) ou la poursuivent quand elle s'échappe de la villa des mafieux. La rage et la haine sont symbolisées par des flammes immenses qui s'échappent du corps de Gino à chaque fois qu'Ulli lui tient tête (392-395). Pour figurer la répulsion qu'inspirent les avances insistantes de l'un des mafieux, Lust l'a dessiné 
comme une substance gluante qui se colle à l'héroïne et prend peu à peu la forme d'un pénis (371-372). Lorsque, traumatisée par le viol dont elle a été victime, Ulli tente de se rendre invisible, son image s'estompe et elle n'apparaît plus que sous la forme d'une silhouette transparente (246-249) ou d'un misérable squelette calciné (278-279). Un flash-back met en scène l'une des histoires qu'Ulli, à douze-treize ans, imaginait pour s'endormir (252-253). Elle imaginait que les hommes capturaient les femmes pour couper leurs corps en morceaux et les exposer dans un musée. Il y a ici, sous forme d'allégorie, une évocation de la fétichisation du corps féminin faite à partir du point de vue féminin.

De telles expérimentations graphiques pour traduire des sentiments jusqu'alors absents de la BD présentent un intérêt non négligeable pour l'évolution du médium. Dans un entretien avec Hugues Dayez (2002), David B. évoquait un vaste champ encore largement à explorer : «Le problème, c'est que la BD ne s'est pas assez confrontée au monde des sentiments et des émotions, elle reste trop souvent dans des canevas très stéréotypés. » Dans sa façon de s'approprier les ressources du médium, Lust nous fait partager la perspective d'une femme sur la société et cherche un langage visuel pour rendre compte d'une expérience du monde différente de celle des hommes.

En se réclamant du féminisme, Catel Muller et Ulli Lust ne politisent pas la bande dessinée de la même manière que des productions militantes aussi radicales que Wimmen's Comix et Ah! Nana des années 1970. Les temps ont changé, le radicalisme suscite aujourd'hui la méfiance et les discours en faveur de l'égalité ont pris d'autres formes. Cet article ne s'attache bien entendu qu'à deux exemples, mais deux exemples révélateurs de notre époque. Ces deux auteures contribuent certes à l'entrée en scène d'une nouvelle génération d'héroïnes au sein du neuvième art, plus complexes, plus ancrées dans le réel. Par le biais du témoignage, elles dénoncent les injustices auxquelles le sexisme ordinaire les expose et décrivent les stratégies que les femmes inventent pour affirmer leur subjectivité. Ces démarches constituent sans aucun doute un tout premier pas, encore très timide, dans le sens d'une légitimité artistique et, partant, peuvent être qualifiées de modérément féministes sur le plan politique. Sur le plan esthétique, cependant, pour que les créatrices de $\mathrm{BD}$ puissent réellement parler d'égale à égal avec les hommes sur un terrain qu'ils occupent majoritairement, il faudra aussi qu'elles inventent vraiment une nouvelle voie, une voix spécifiquement féminine, c'est-à-dire de nouveaux modes d'expression pour traduire une modalité du sentir entièrement différente de celle des hommes, une façon différente d'envisager les ressources expressives de la bande dessinée. Pour imiter la célèbre formule du marquis de Sade : auteures, encore un effort! 


\section{Bibliographie}

Anonyme. «Héro(ine)s » au Lyon BD Festival. 3-27 juin 2015. Web. 6 juin 2015.

Anonyme. «Les vingt ans de Catel.» L'Etudiant. juillet-août 2015. Web. 6 juin 2015.

Alféef, Emmanuelle. «Peu de femmes dans la BD, mais pas de machisme. » L'Express, 5 août 2011. Web. 6 juin 2015.

Association Artémisia. Interview de Chantal Montellier. wnw.associationartemisia.wordpress.com. Web. 6 juin 2015.

Berkani, Véronique, «Catel Muller et les clandestines de l'Histoire. » l'Alsace, 29 décembre 2014. Web. 6 juin 2015.

Muller, Catel. Site officiel de Catel Muller. Web. 18 décembre 2015

Muller, Catel et Louis Bocquet. Kiki de Montparnasse. Paris : Casterman, 2007. . Ainsi soit Benoite Groult. Paris : Grasset, 2014.

Charte des créatrices de bande dessinée contre le sexisme, septembre 2015. Web. 6 juin 2015.

Cixous, Hélène. Le Rire de la Méduse. Paris : L'Arc, 1975

Collectif. «Hire more women - here's a few to get you started. » pétition en ligne. Web. 6 juin 2015.

Dayez, Hugues. La Nouvelle bande dessinée, Blain, Blutch, David B., de Crécy, Dupuy-Berberian, Guibert, Rabaté, Sfar. Bruxelles : Niffle, 2002.

Evans, Christophe, et Françoise Gaudet. «La lecture de bandes dessinées. » Culture études 2.2 (2012) : 1-8.

Guilbert, Xavier. "La légitimation en devenir de la bande dessinée.» Comicalités. 17 mai 2011. Web. 6 juin 2015.

Groensteen, Thierry. «Les paradoxes de la BD au féminin. » Neuf et demi, 15 janvier 2010. Web. 6 juin 2015.

Kempinaire, Erik (propos recueillis par). «Catel et Bocquet : "Kiki fait partie de l'imaginaire collectif, comme le poster de Che Guevara".» ActuaBD, 19 avril 2007. Web. 6 juin 2015.

Lestavel, François. «Catel \& Bocquet tutoient l'Olympe. » Paris Match, 12/18 avril 2012. Web. 6 juin 2015.

Lust, Ulli. Fashionvictims - Trendverächter: Bildkolumnen und Minireportagen aus Berlin. Berlin : Avant-Verlag, 2008.

. Air Pussy. Electrocomics. Web. 6 juin 2015.

. Heute ist der leţte Tag vom rest deines Lebens. Berlin : Avant-Verlag, 2009.

Trop n'est pas assez (trad. Jörg Stickan). Bussy-Saint-Georges : Çà et là, 2012.

Médioni, Gilles et Marion Festraëts. dossier « La BD au féminin. »L'Express, 27 janvier 2009. Web. 6 juin 2015.

Miller, Ann. «Citizenship and City Spaces.» Mark McKinney (ed.). History and Politics in French-Language Comics and Graphic Novels. Jackson: University Press of Mississippi, 2008.

Mulvey, Laura. "Visual Pleasure and Narrative Cinema.» Screen 16:3 
(Autumn 1975):6-18. 6 juin 2015.

Quillet, Lucie. «BD : comment les femmes sont passées de Bécassine à Super Woman. »,Figaro Madame, 29 janvier 2015. Web. 6 juin 2015.

Quillien, Christophe. Elles, grandes aventurières et femmes fatales de la bande dessinée. Paris : Éditions Huginn \& Muninn, 2015.

Ratier, Gilles. «Sur la production d'une année de bande dessinée dans l'espace francophone européen. » 2015. Web. 6 juin 2015.

Reyns-Chikuma, Chris. "Entretien avec Catel Muller », Alternative francophone 1:7 (2014) : 71-85. Web. 6 juin 2015.

Sobel, Marc. «The Ulli Lust Interview. » The Comics Journal. 3 janvier 2003. Web.

Tanxxx. «Les pétasses, l'abêtissement et les éditeurs. » Des croûtes aux coins des yeux. 30 août 2011. Web. . « Auto-droit de réponse. » 8 juillet 2015. Web. 6 juin 2015. 